Supporting Information

\title{
Haptotropic Metal Migration in Densely Substituted Hydroquinoid Phenanthrene $\mathrm{Cr}(\mathrm{CO})_{3}$ Complexes
}

\author{
Karl Heinz Dötz, ${ }^{,}{ }^{\dagger}$ Joachim Stendel jr., ${ }^{\dagger}$ Sonja Müller, ${ }^{\dagger}$ Martin Nieger, ${ }^{\ddagger}$ \\ Sombat Ketrat, ${ }^{\S}$ Michael Dolg**,
}

Kekulé-Institut für Organische Chemie und Biochemie, Rheinische FriedrichWilhelms Universität Bonn, Gerhard-Domagk-Strasse 1, D-53121 Bonn, Germany, Institut für Anorganische Chemie, Rheinische Friedrich-Wilhelms Universität Bonn, Gerhard-Domagk-Strasse 1, D-53121 Bonn, Germany, and Institut für Theoretische Chemie, Universität zu Köln, Greinstrasse 4, D-50939 Köln, Germany

\footnotetext{
* Author to whom correspondence should be addressed. Fax: Int.+49 228 735813. E-mail: doetz@unibonn.de.

** Author to whom correspondence regarding DFT calculations should be addressed. Fax: Int.+49 221 470 6896. E-mail: m.dolg@uni-koeln.de.

${ }^{\dagger}$ Kekulé-Institut für Organische Chemie und Biochemie.

${ }^{\ddagger}$ Institut für Anorganische Chemie.

$\S$ Institut für Theoretische Chemie.
} 
NMR spectra of rearranged phenanthrene chromium tricarbonyl complexes

\section{5-20 and 22}

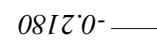

$1990^{\circ} 0^{-}$
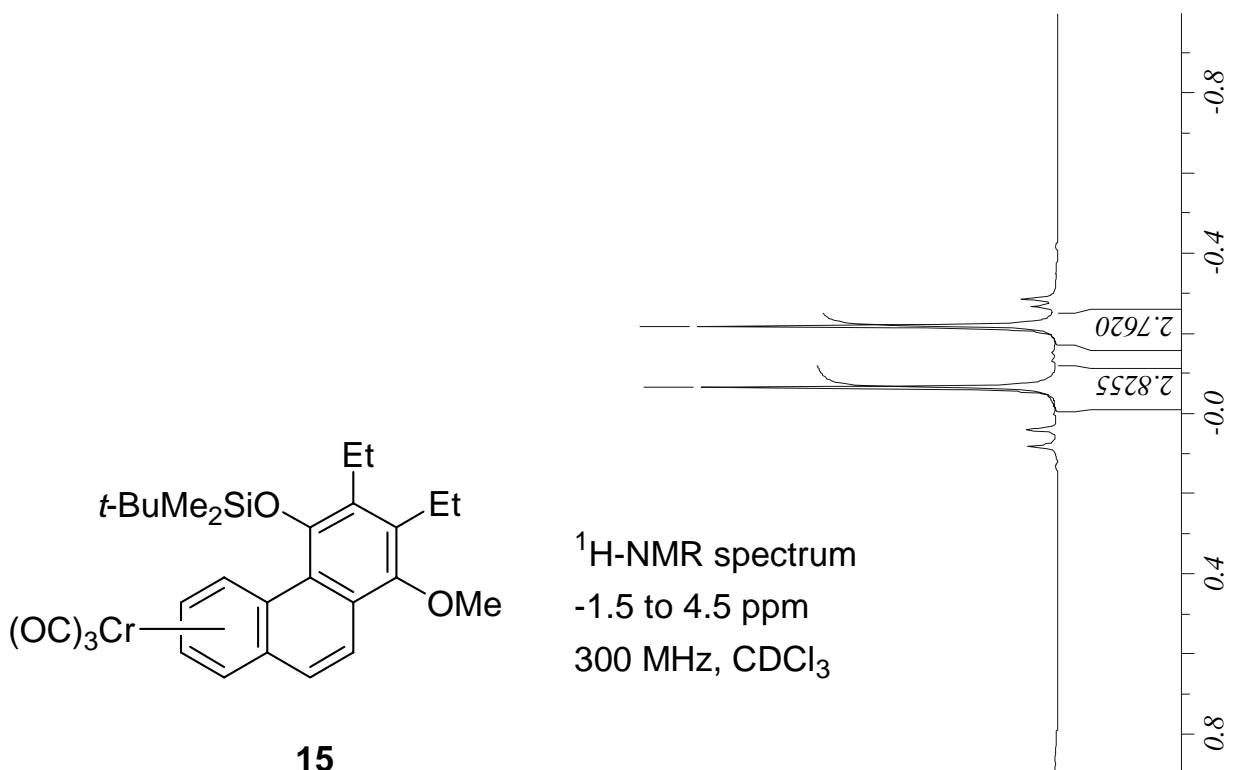

15

$99 S I^{\circ} I$

$6 I E Z^{\prime} I$

$0 \angle S Z^{\circ} I$

$S I 8 C^{\circ} I$

${ }^{1} \mathrm{H}$-NMR spectrum

-1.5 to $4.5 \mathrm{ppm}$

$300 \mathrm{MHz}, \mathrm{CDCl}_{3}$

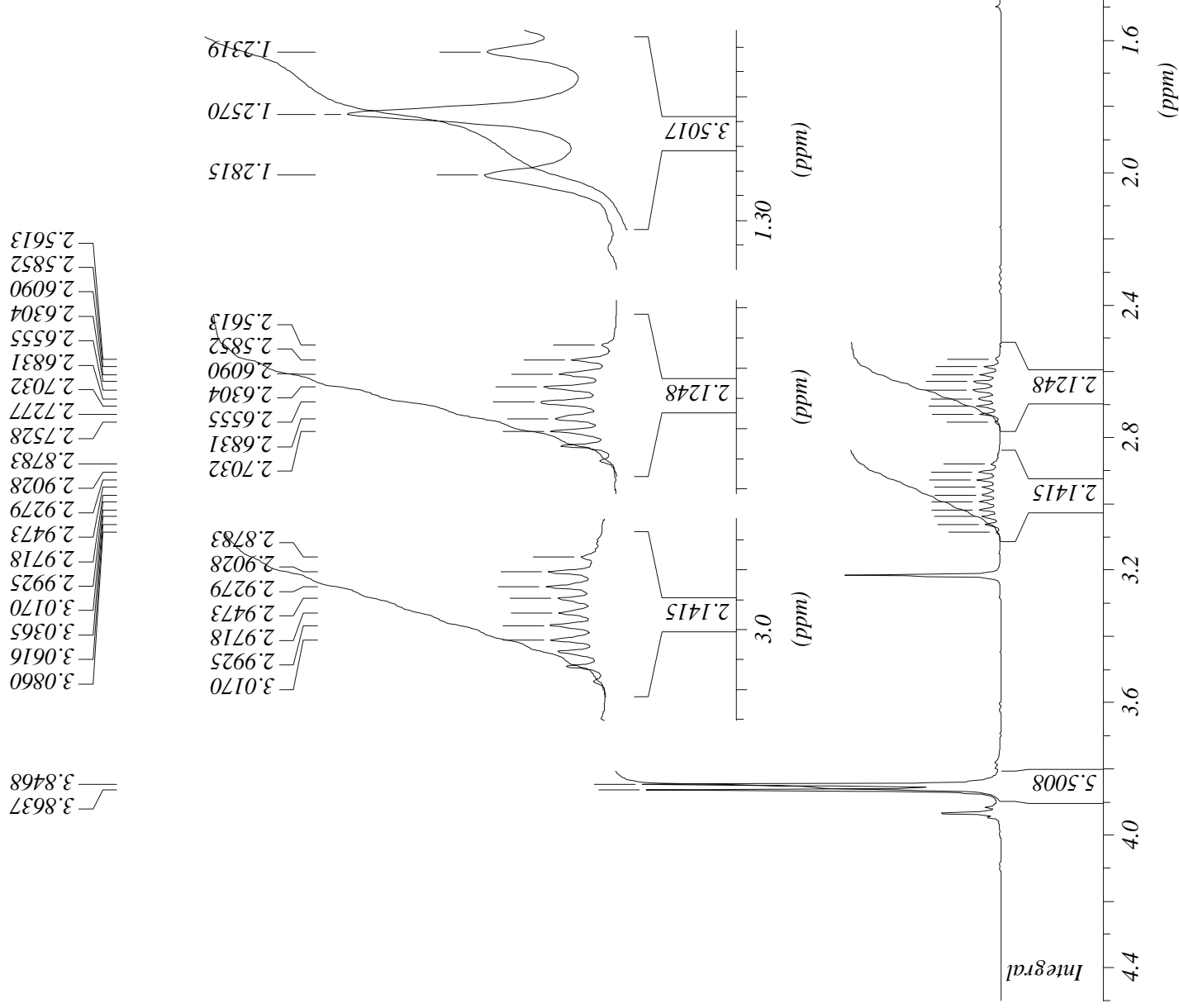



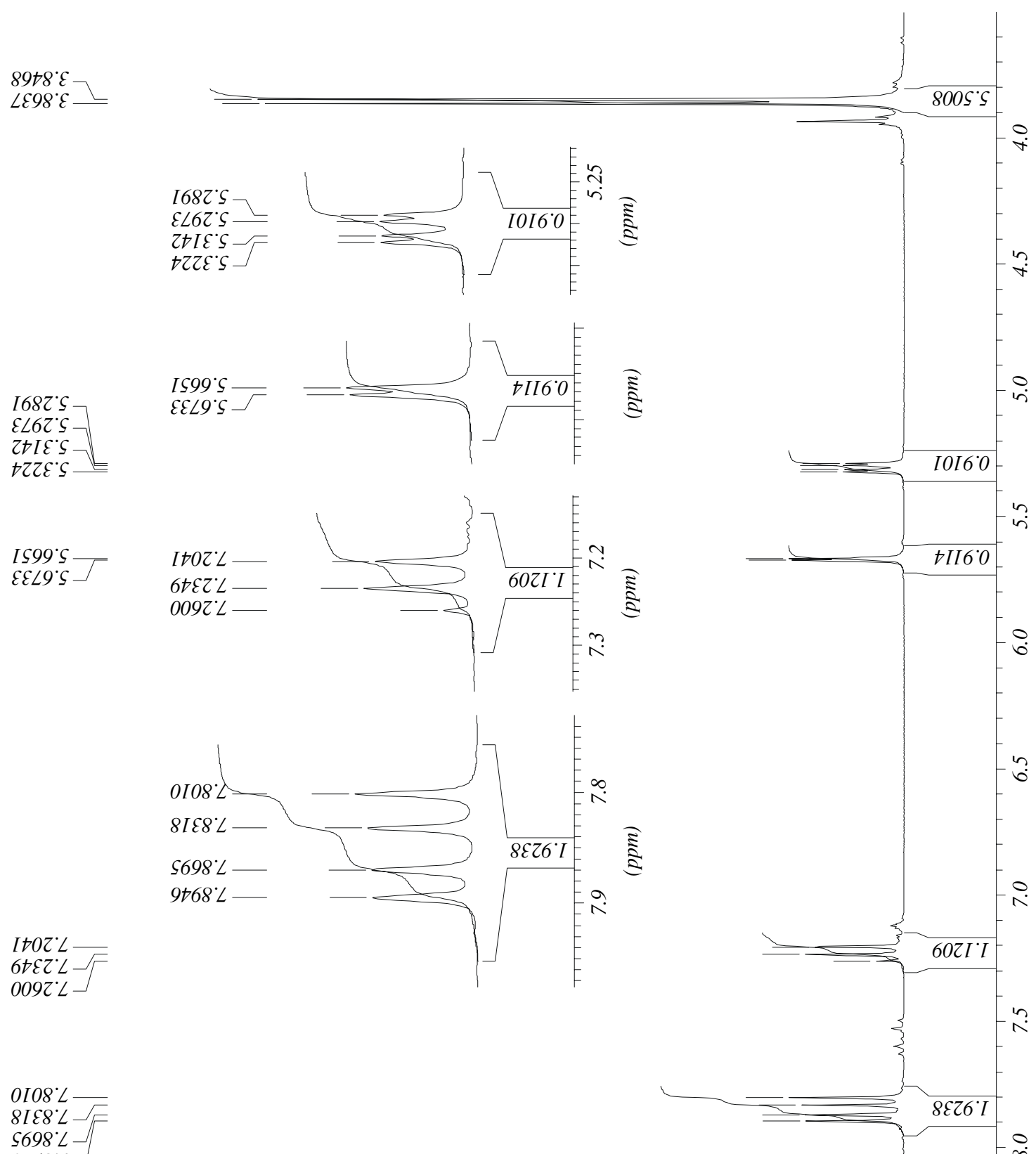

3

$9+68^{\circ}$<smiles>CCc1c(OC)c(OC)c2ccc3cc(Cl)ccc3c2c1OCC(C)(C)C</smiles>

15
${ }^{1} \mathrm{H}-\mathrm{NMR}$ spectrum

3.5 to $10.0 \mathrm{ppm}$ $300 \mathrm{MHz}, \mathrm{CDCl}_{3}$ 
$06588^{\circ}$

$5989^{\circ} \mathrm{Z}^{-}$

ItII'SI

668S"SI

$\angle D O D^{\circ} 8 \mathrm{I}$

$0 \angle S I^{\circ} 0 Z$

$\angle \triangleright 80^{\circ} \mathrm{LZ}$

OL96"SZ

90IL'SS

$\pitchfork 8 \angle 8 \div 2$

$0986{ }^{\circ} \mathrm{DL}$

tSS8 84

$6902^{\circ} \varepsilon 6$

ES8I:66 -

$\triangle \nrightarrow S 0^{\circ} \mathrm{EOI}$

Z†OZ'LCI

$966 \varepsilon^{\circ} \nabla Z I$

90IS" $\pitchfork Z I$

E6tS'SZI

I6I9 $S E L$

$60 \varepsilon 5^{\circ} 9 \varepsilon I$

SLZ8'ItI

$260 I^{\circ} 9 t \mathrm{t}$

$6 S 09^{\circ} 6 t I$

Z†OZ'IZI -

$966 \varepsilon^{\circ} \nabla Z I$

90IS" $๐ 2 I=$

E6tS $S Z I$

60ES $981-$

$S \angle Z 8^{\circ} I D I$

$260 I^{\circ} 9 t$

$6509^{\circ} 6 t$

$S \angle L I^{\circ} E E Z$<smiles></smiles>

${ }^{13} \mathrm{C}-\mathrm{NMR}$ spectrum

$75 \mathrm{MHz} \mathrm{CDCl}_{3}$

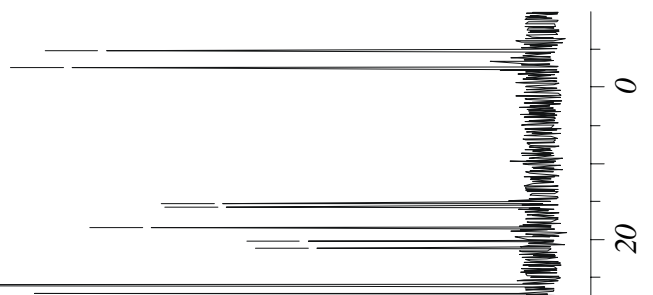


<smiles></smiles>

16

$8+0 Z^{\circ} 0^{-}$

$\stackrel{+}{i}$

$S E S 0^{\circ} 0^{-}$

S89 I $T$

$98 \angle I^{\circ} I$

$0 E 8 I^{\circ} I$

$S \angle O Z^{*} I$

$6 I E Z^{\circ} I$

$S \sqcup \nabla Z^{\prime} I$

$969 Z^{\circ} I$

$\angle \triangleright 6 Z^{\circ} I$

\section{6}
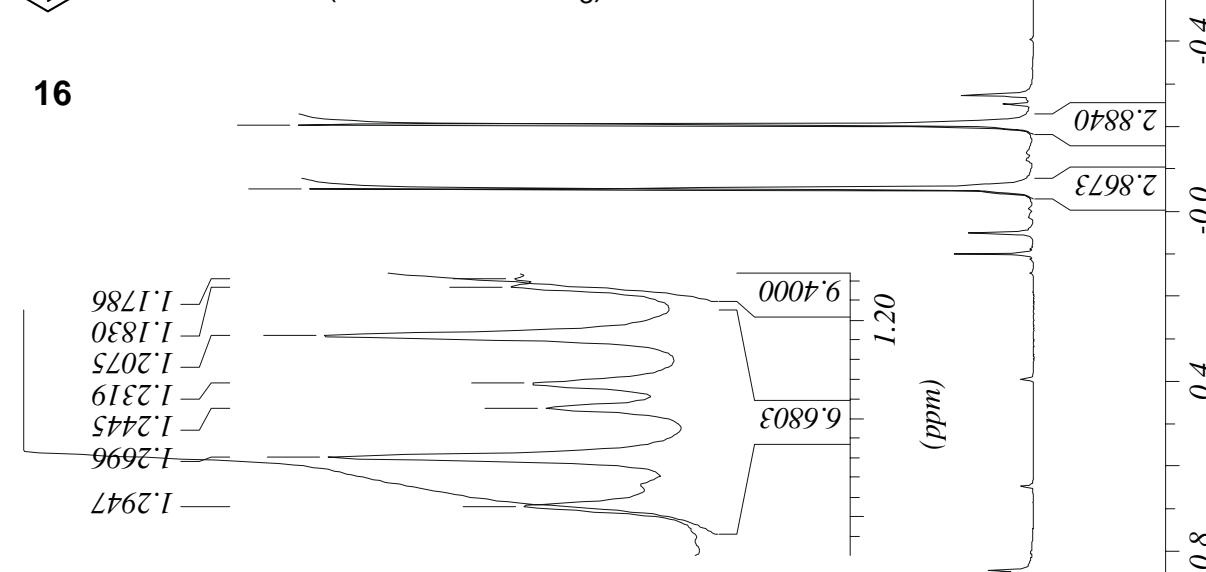

$\stackrel{0}{1}$

$\stackrel{8}{0}$

$\stackrel{\infty}{\circ}$

$\angle \forall Z 9^{\circ} \mathrm{Z}$

$8+t 9^{\circ} Z$

$6699 \cdot z$

$9 S 69^{\circ} \mathrm{Z}$

$\angle S I L^{\prime} Z$

$\nabla 9 \angle S^{\circ} Z$

$2009^{\circ} \mathrm{Z}$

$\angle \Delta Z 9^{\circ} Z$

$8+t 9^{\circ} Z$

$6699^{\circ} \mathrm{Z}$

$9 S 69^{\circ} \mathrm{Z}$

$\angle S I \angle \cdot Z$

$Z 0 t \angle \cdot Z$

$\varepsilon S 9 \angle ' Z$

$\downarrow \varepsilon 68^{\circ} Z$

$8 \angle I 6^{\circ} Z$

$6 Z \nabla 6^{\circ} \mathrm{C}$

$\downarrow Z 96^{\circ} Z$

$6986^{\circ} \mathrm{Z}$

$9 \angle 00^{\circ} \mathcal{E}$

$\angle Z \varepsilon 0^{\circ} \varepsilon$

$Z Z S 0^{\circ} \mathcal{E}$

$\varepsilon \angle \angle 0^{\circ} \varepsilon-$

$\angle$ IOI $^{\circ} \mathrm{E}-$

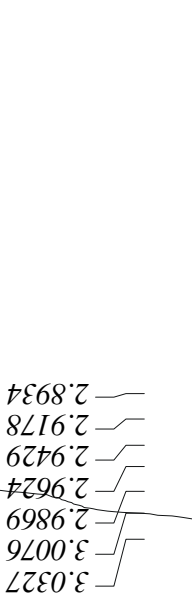

$\angle Z E 0^{\circ} \varepsilon$

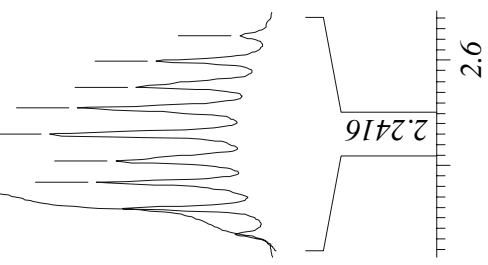

ป

$\stackrel{\circ}{\sim}$

$\stackrel{\nabla}{i}$

$9 L \tau^{\prime} Z$

$\stackrel{\infty}{\text { v }}$

$\rightarrow \angle t 8^{\circ} \mathcal{E}$

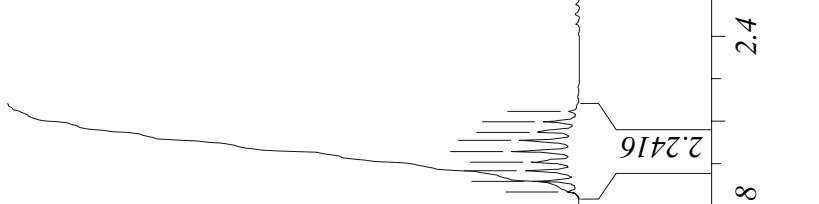

IE $\angle 8^{\circ} \mathcal{E}$

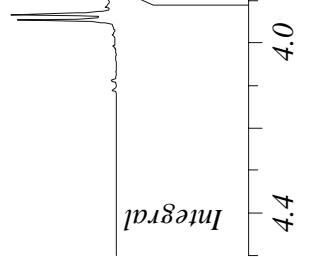




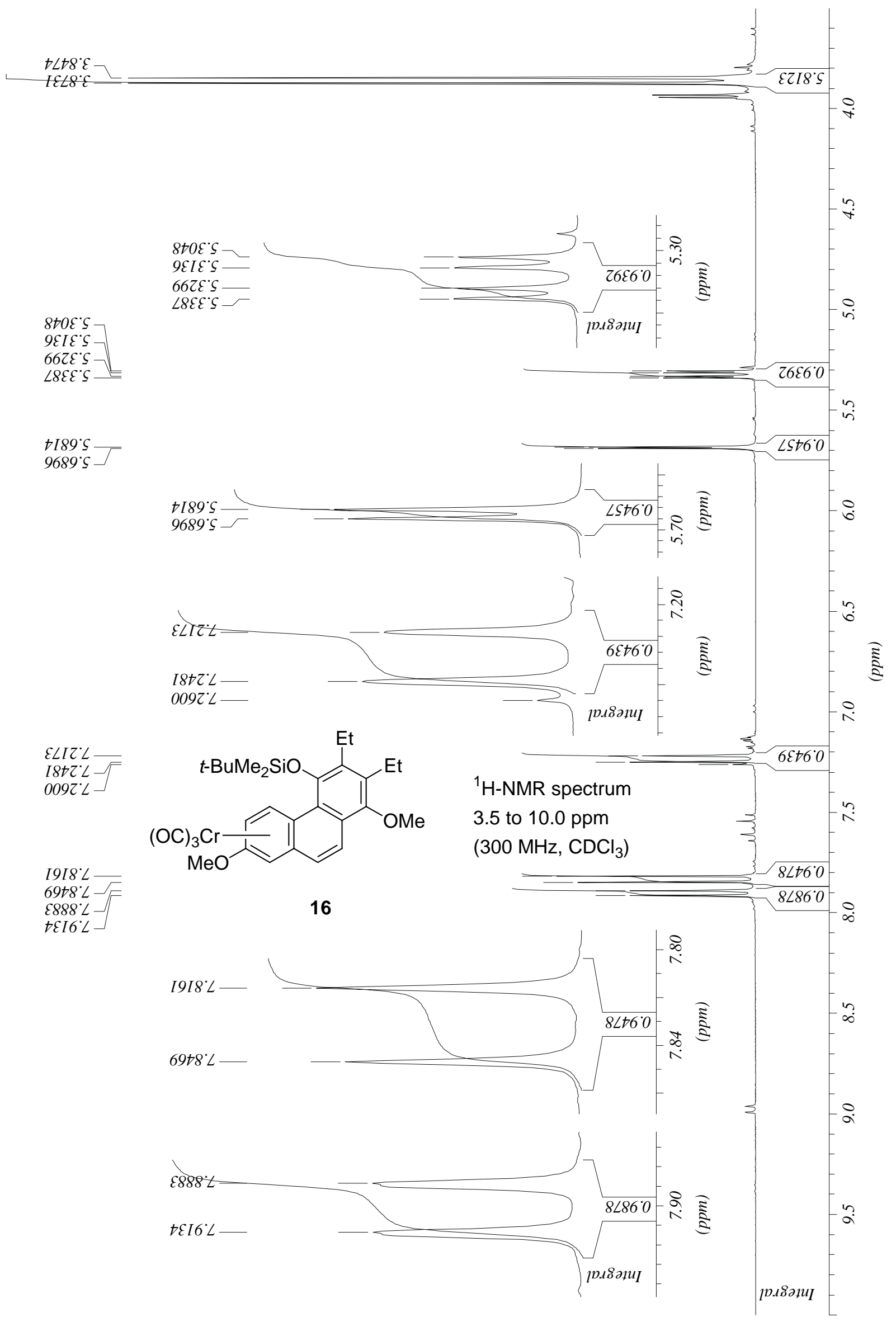


$9868^{\circ} t$

$\mathrm{Z} 8 \mathrm{~L} / \mathrm{L} \cdot \mathrm{-}$

$\neg Z 80^{\circ} \mathrm{SI}$

I99S $S I$

OS9E: $8 I$

ESZI $0 Z$

OESO ${ }^{\circ} \mathrm{LZ}$

$\varepsilon 676^{\circ} \mathrm{SZ}$

OL $\angle 9{ }^{\circ} S S$

6088.79

$0+66^{\circ}+\angle$

$0000^{\circ} \angle L$

tSS8 84

$066 I^{\circ} \varepsilon 6$

9ESI*66

$9290^{\circ} \mathrm{EOL}$

St9 $I^{\circ} I Z I-$

8SLE" $\neg Z I-F$

898t ${ }^{\circ} \mathrm{CL} I$

$\downarrow \varepsilon E S S^{\circ} S Z I$

S6LS'SEI - F

EL6t"9EI

$\angle I L 8 \cdot I t I-$

$\downarrow S 80^{\circ} 9 t I$

IZ85"6力I -

$969 I^{\circ} \varepsilon E Z$
$S \pitchfork 9 I^{\circ} I Z I$

8SLE'tZI

$898 t \rightarrow Z I-$

$E E S S^{\circ} S Z I$

S6LG'SEL

EI6t $9 \varepsilon 1-$

$\angle I I 8{ }^{\circ}$ It

$\downarrow S 80^{\circ} 9 t \mathrm{~L}$

IZ8S' $6 t$ I

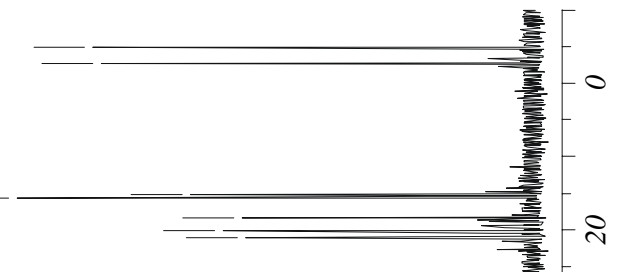

<smiles></smiles>

${ }^{13} \mathrm{C}$-NMR spectrum ( $75 \mathrm{MHz}, \mathrm{CDCl}_{3}$ ) 


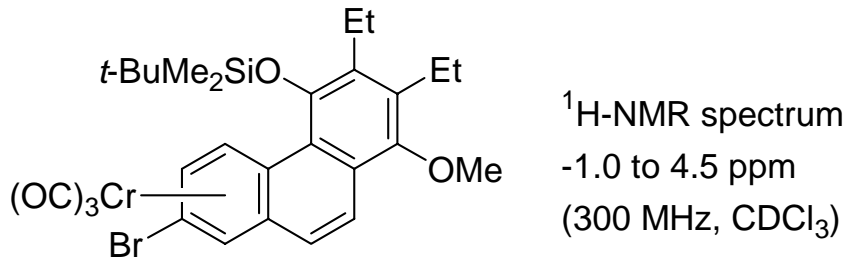

EZEQ' $Z$

$688 S^{\circ} \mathrm{Z}$

$966 \varsigma^{\prime} \mathrm{Z}$

$8 \angle 09^{\circ} \mathrm{Z}$

$\varepsilon Z E 9^{\circ} Z$

$\angle I S 9^{\circ} Z$

95292

$+669^{\circ} \mathrm{Z}$
$68 \mathrm{~L} L \mathrm{~L}$

$\angle I S 9^{\circ}$

$9 S \angle 9^{\circ} Z$

$\$ 669^{\circ} \mathrm{Z}$

$68 I L ' Z$

$S 6 Z L ' Z$

$0 t t \angle ' Z$

$589 L^{\prime} \mathrm{Z}$

S6ZL' $Z$

$0+t \angle \cdot z$

$589 L ' Z$

$92 \angle 8^{\circ} \mathrm{Z}$

$6 \varepsilon 88^{\circ} \mathrm{Z}$

$8468^{\circ} \mathrm{Z}$

$0606^{\circ} \mathrm{Z}$

It $26^{\circ} \mathrm{Z}$

$26+6^{\circ} \mathrm{Z}$

$\angle 896^{\circ} \mathrm{Z}$

$8 \varepsilon 66^{\circ} \mathrm{Z}$

$\angle O I 0^{\circ} \mathcal{E}$

$Z S \mathcal{L} 0^{\circ} \mathcal{E}$

$\angle t S 0^{\circ} \mathcal{E}$

$92 \angle 8^{\circ} \mathrm{Z}$

$6 \mathcal{6 \varepsilon 8 ^ { \circ } \mathrm { Z }}$

$8 \angle 68^{\circ} \mathrm{Z}$

06062

$I+26^{\circ} \mathrm{Z}$

$26+6.2$

$\angle 896^{\circ} \mathrm{Z}$

$8 \mathcal{E} 66^{\circ} \mathrm{Z}$

$\angle 0 I 0^{\circ} \mathcal{E}$

$\tau \varsigma \mathcal{E} 0^{\circ} \mathcal{E}$

$\angle t S 0^{\circ} \mathcal{E}$

$86 \angle 0^{\circ} \mathcal{E}$

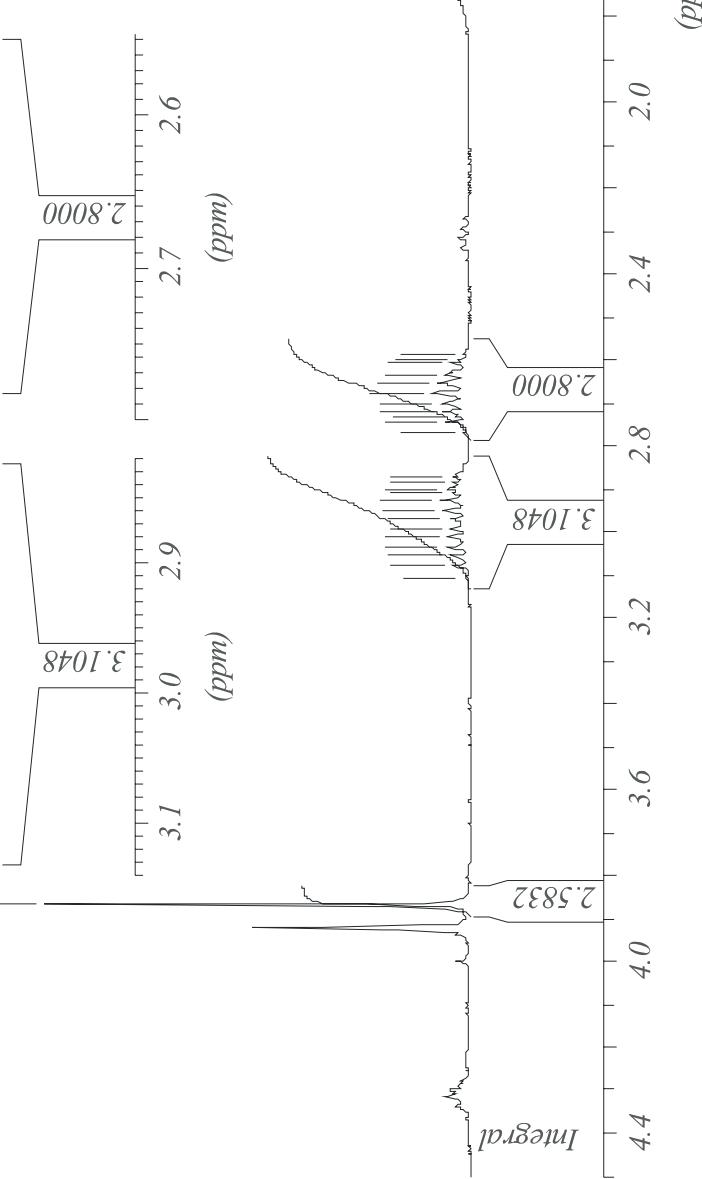


<smiles>CCc1c(OC)c(OC)c2ccc3cc(Br)ccc3c2c1OCC(C)(C)C</smiles>

${ }^{1} \mathrm{H}$-NMR spectrum

3.5 to $10.0 \mathrm{ppm}$

(300 MHz, $\mathrm{CDCl}_{3}$ )

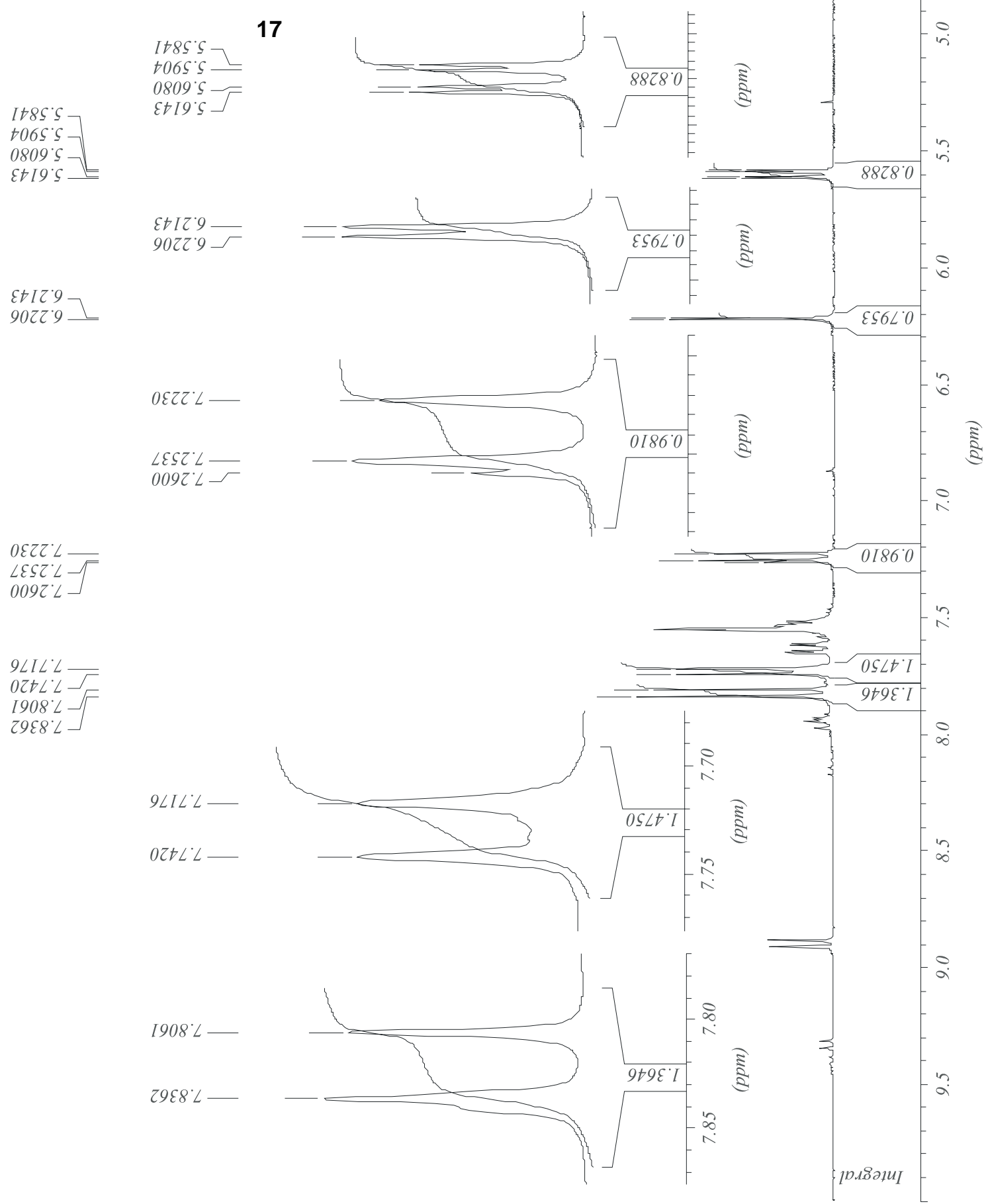


Z8IL $Z^{-}$

$+2 \oplus \tau^{\circ} \mathrm{C}-$

$9266^{\circ} 0$

$6869^{\circ} \circ \mathrm{I}$

$87+0^{\circ} s I$

$t \leftarrow \varepsilon S \subseteq I$

$0 \mathcal{E} \angle \mathcal{E} 8 I$

9S $\angle 66^{\circ} I$

ISLT $6 I$

SZIZ:0Z

$89 \angle 0^{\circ} \mathrm{IZ}$

$6519^{\circ} \mathrm{IZ}$

$\varsigma 208^{\circ} \varsigma Z$

SEI ${ }^{\circ} \mathrm{SZ}$

$8 I \angle 9^{\circ} 6 Z$

$\angle 8 \angle t^{\circ} Z \mathcal{E}$

IOZE $t \mathcal{E}$

$06 T^{\circ} 9 \mathcal{E}$

I816 29

$\varepsilon \angle t Z^{\circ} \angle 9$

$0000^{\circ} \mathrm{LL}$

9ZEZ I6

$8+\angle 8^{\circ} I 6$

$9889^{\circ} 76$

$6+00: \mathrm{L6}$

Et86 I0I

9LEL'ZOI

$9+I \varsigma^{\circ} \mathcal{E O I}$

$\angle 6 E 8^{\circ} \mathcal{E} 0 I$

$9827^{\circ}$ ZII

$8566^{\circ} 021$

$898 t^{\circ}+2 I$

S8IS ${ }^{\circ} Z I$

6LZL $6 Z I$

$\angle S 6 I^{\circ} 0 E I$

Z908.0EI

8ES ${ }^{\circ} 0 \mathrm{EI}$

$t S I I \cdot I \mathcal{~} I$

$\varsigma \subseteq \angle t 9 \varepsilon I$

6I $\angle 8^{\circ} 9 \mathcal{E} I$

zztt $9 t I$

$\angle I Z 9^{\circ} 6 t I$
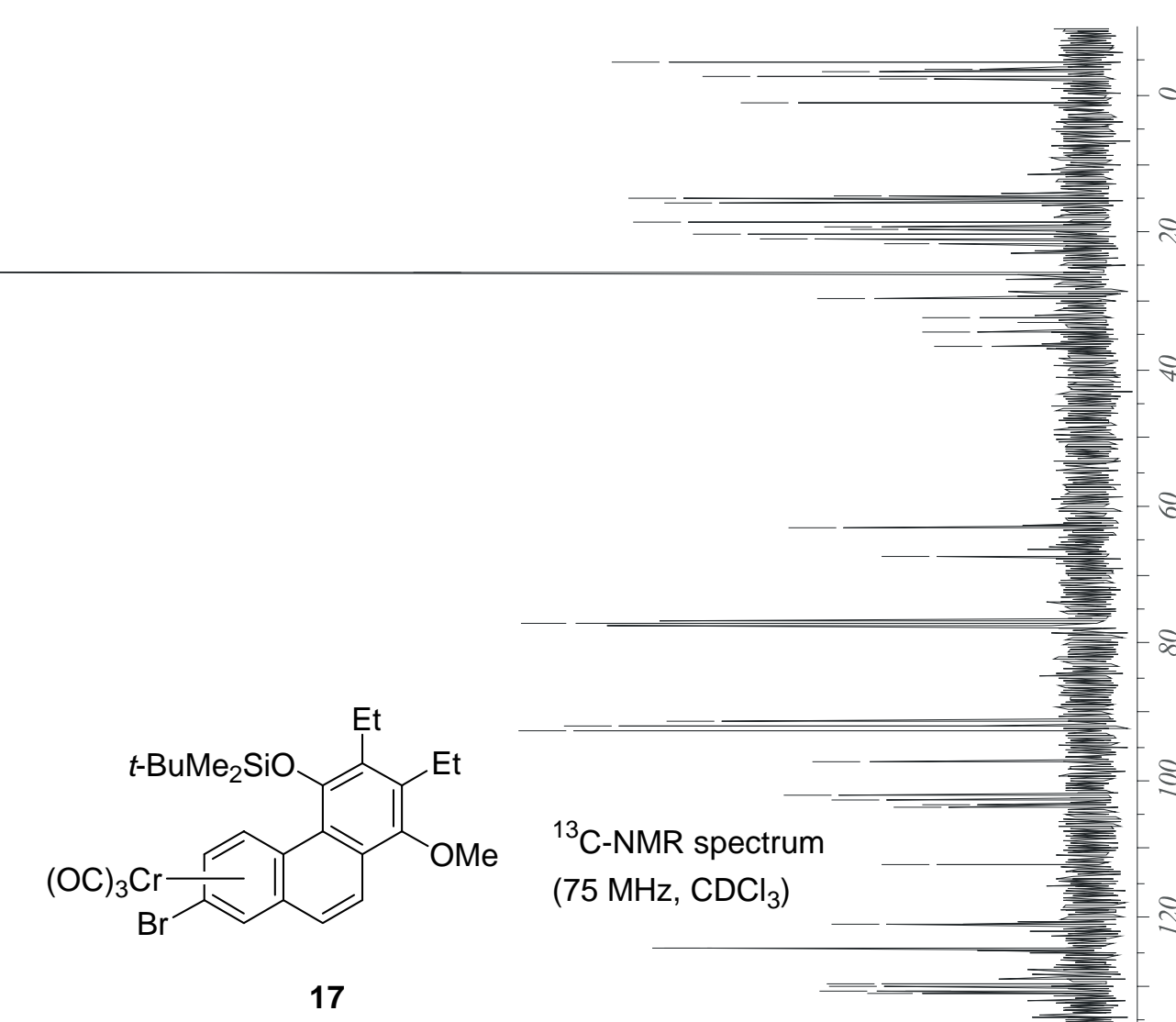

○

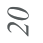

$-\gtrless$

$-8$

$-\infty$

8

${ }^{13} \mathrm{C}-\mathrm{NMR}$ spectrum

(75 MHz, $\mathrm{CDCl}_{3}$ )

$\stackrel{\vartheta}{\searrow}$

ำ

9zEน 16

$8+\angle 8 \cdot 16$

9889'76-

$6+00 \div 6$

Et86:I0I

9LEL'ZOI

$9+I \mathcal{C}^{\circ} \mathcal{E} O I$

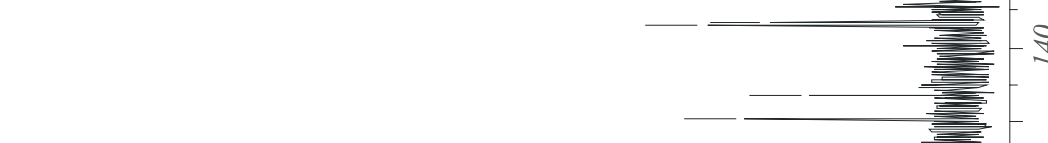

$\stackrel{8}{8}$

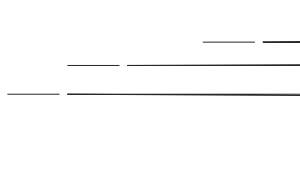

ส

$\infty$

ह

6SLS'IEZ -

$\angle \mathcal{E} 8 \mathcal{E} E \mathcal{E}$

6LZLOCZI

$\angle S G I^{\circ} 0 E I$

z908.0EI

8ES $8^{\circ} 0 \mathcal{E} I$

$t S I I^{\circ} I E I$

$S \varsigma \angle T^{\circ} 9 \mathcal{E} L$ 6I $\angle 8^{\circ} 9 E I$

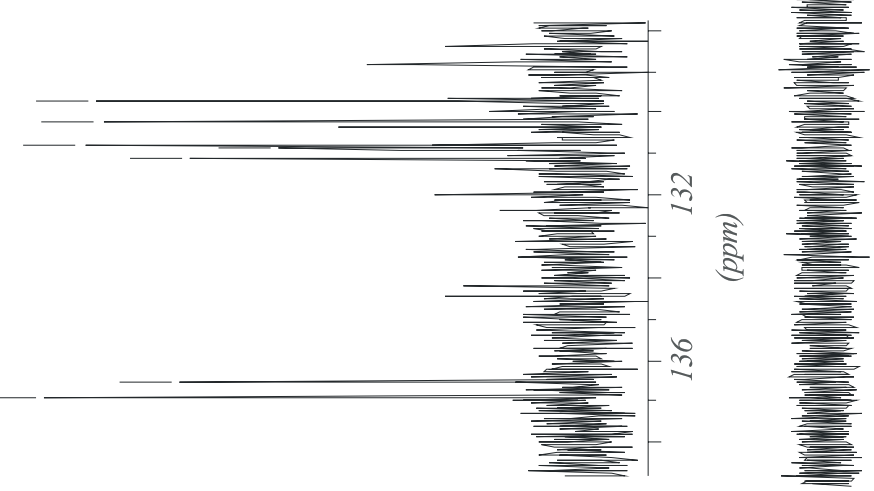

$\stackrel{ }{\sim}$

$\stackrel{ }{7}$

$\underset{\infty}{\infty}$ 
<smiles>CCOc1c(CC)c(OC)c2c(ccc3cc(Cl)ccc32)c1OCCOC</smiles>

${ }^{1} \mathrm{H}-\mathrm{NMR}$ spectrum

-1.5 to $4.5 \mathrm{ppm}$

(300 MHz, $\mathrm{CDCl}_{3}$ )

$2866^{\circ} \mathrm{C}$ $6+58^{\circ} \mathcal{E}$
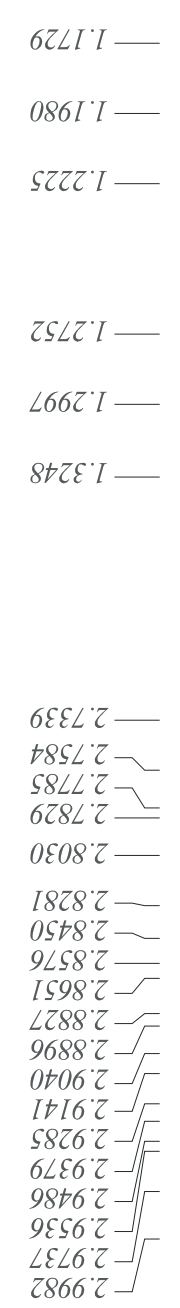

$Z S L Z \cdot I$

$\angle 662^{\circ} I$

$8+\tau \mathcal{E} \cdot I$

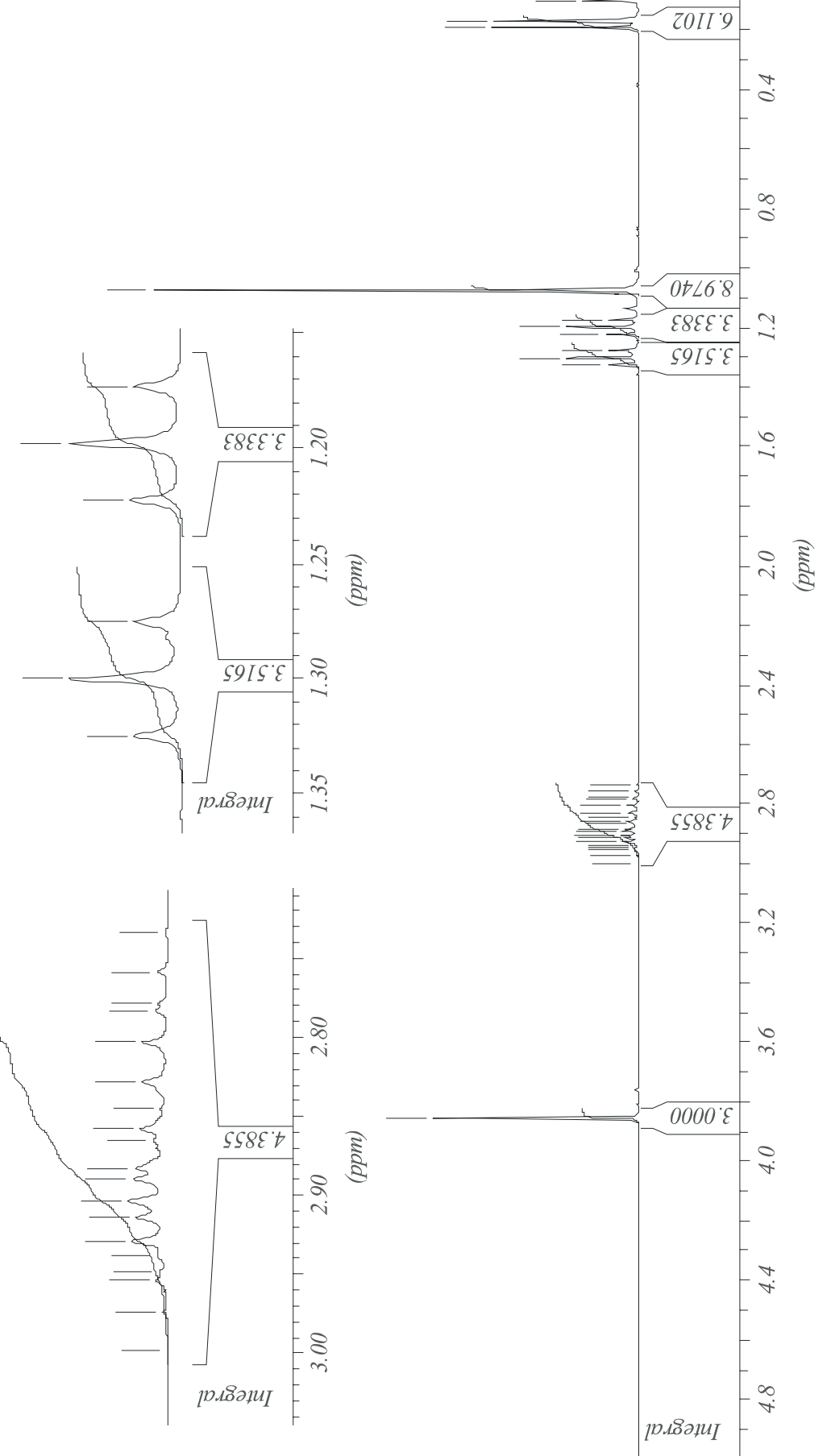



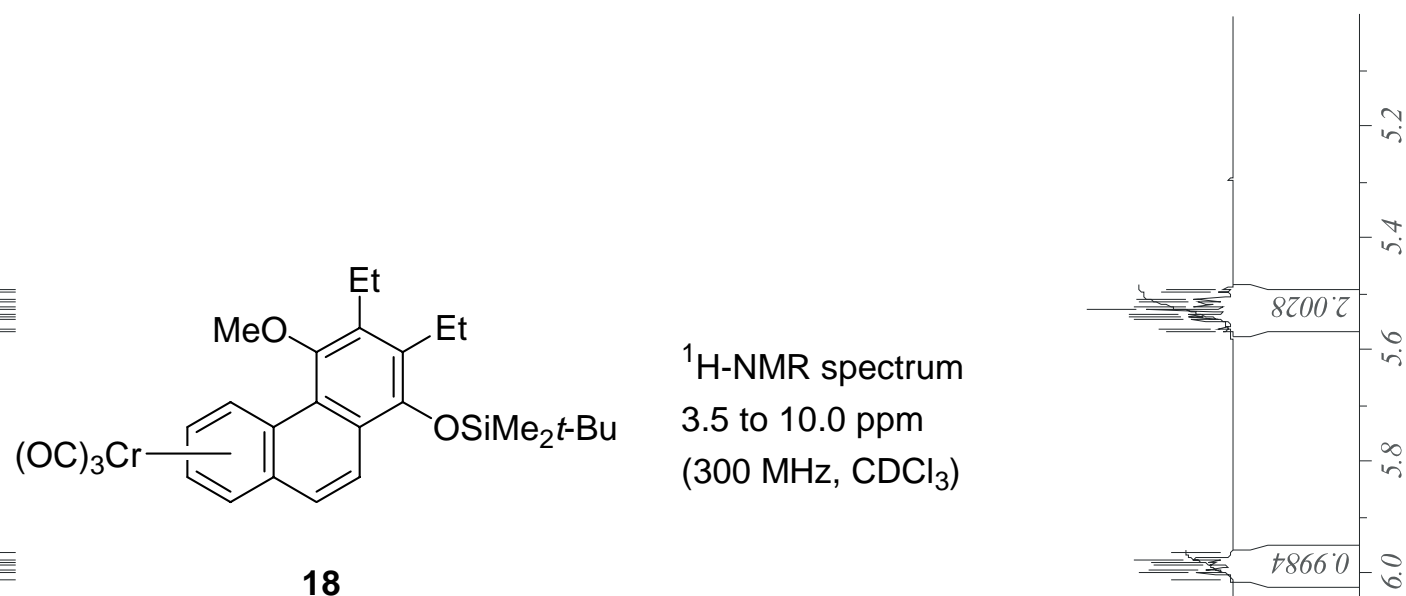

$8086^{\circ} \mathrm{S}$

18

$9+86^{\circ} \mathrm{S}$

$9+66^{\circ} \mathrm{S}$

$5100^{\circ} 9$

SEI0'9

$0+9 I^{\circ} \mathrm{L}$

$006 t^{\circ} \mathrm{S}$

9S6t' $S$

IOIS 'S

t9IS'S

tIIS S

S6ZS'S

E8ES S

St96 S

ZSL6 $S$

$8086^{\circ} \mathrm{S}-$

$9+86^{\circ} \mathrm{S}$

$9+66^{\circ} \mathrm{S}$

SI00 9

SEI0'

$0+9 I^{\circ} \mathrm{L}$

It $6 I^{\circ} \mathrm{L}$

$0092^{\circ} \mathrm{L}$

$I+6 I^{\circ} \mathrm{L}$

$6 S \angle L L$

$728 L^{\circ} \mathrm{L}$

$096 \angle \mathrm{L}$

$\angle 108^{\circ} \mathrm{L}$

$0818^{\circ} \mathrm{L}$

$\angle 8+8^{\circ} \mathrm{L}$

$6 S \angle L L$

$278 L^{\circ} \mathrm{L}$

$096 L \mathrm{~L}$

$\angle I 08^{\circ} \mathrm{L}$

$0818^{\circ} \mathrm{L}$
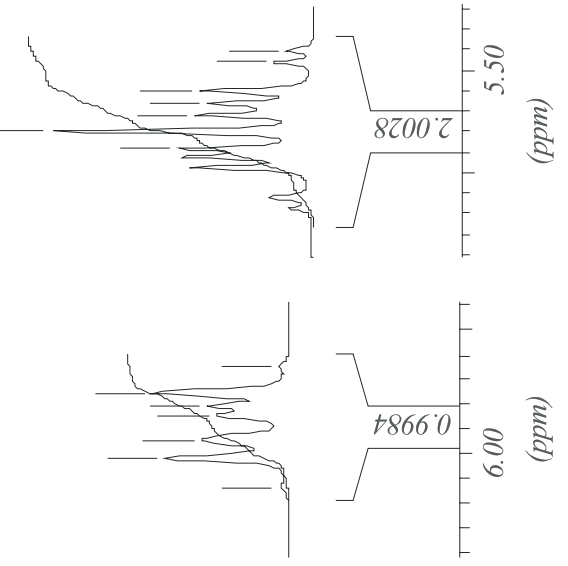

ชี

范

రి

$\infty$

$\therefore \frac{2}{2}$

$+8660^{\circ}$ ह
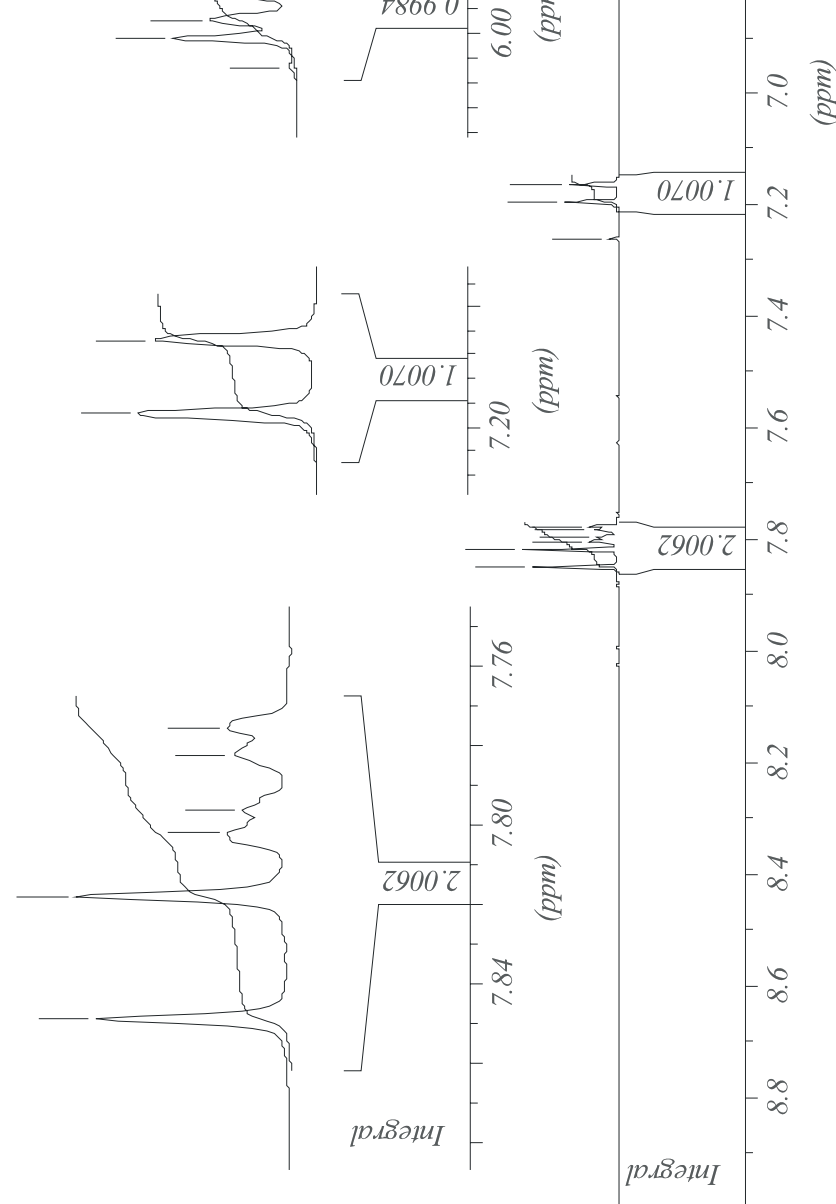
$8 Z I \mathcal{E} \mathcal{E}^{-}$

$\angle L I Z^{\circ} \mathcal{E}^{-}$

$8865^{\circ} \circ I$

$6118^{\circ} S I$

$090 \angle 8 I$

$\angle 0 t^{\circ} 0 Z$

E0t0.07

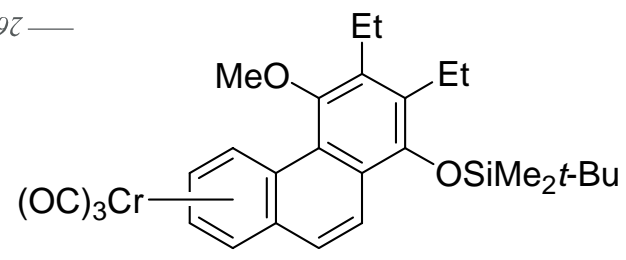

9z25.19

18

$0000^{\circ} \mathrm{LL}$

$\angle 0 S S^{\circ} 06$

$6998^{\circ} 16$

$0296^{\circ} 16$

$9+95^{\circ} 26$

ISIO $\mathrm{E} \mathcal{E} O I$

$\angle 9 L Z^{\circ} \mathcal{E} O I$

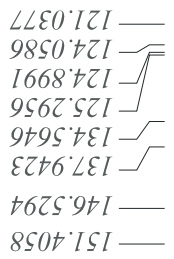

8EZ6 $2 \mathcal{E}$

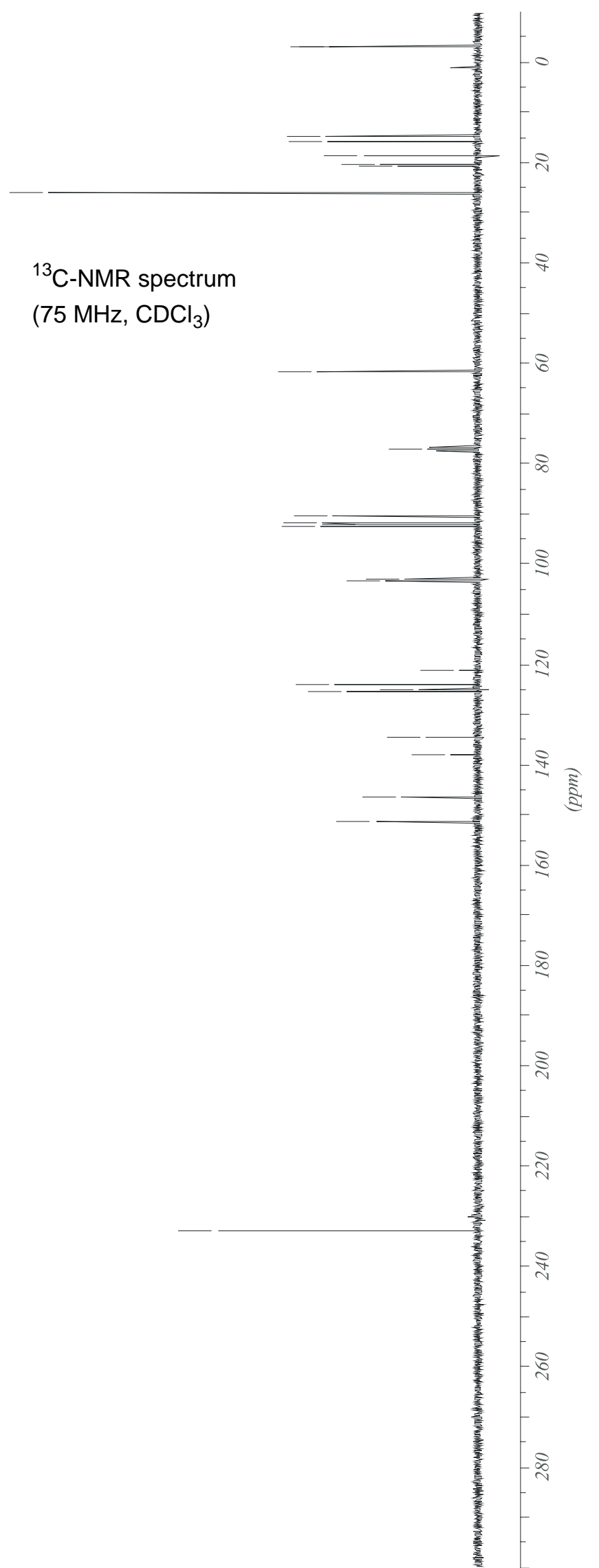



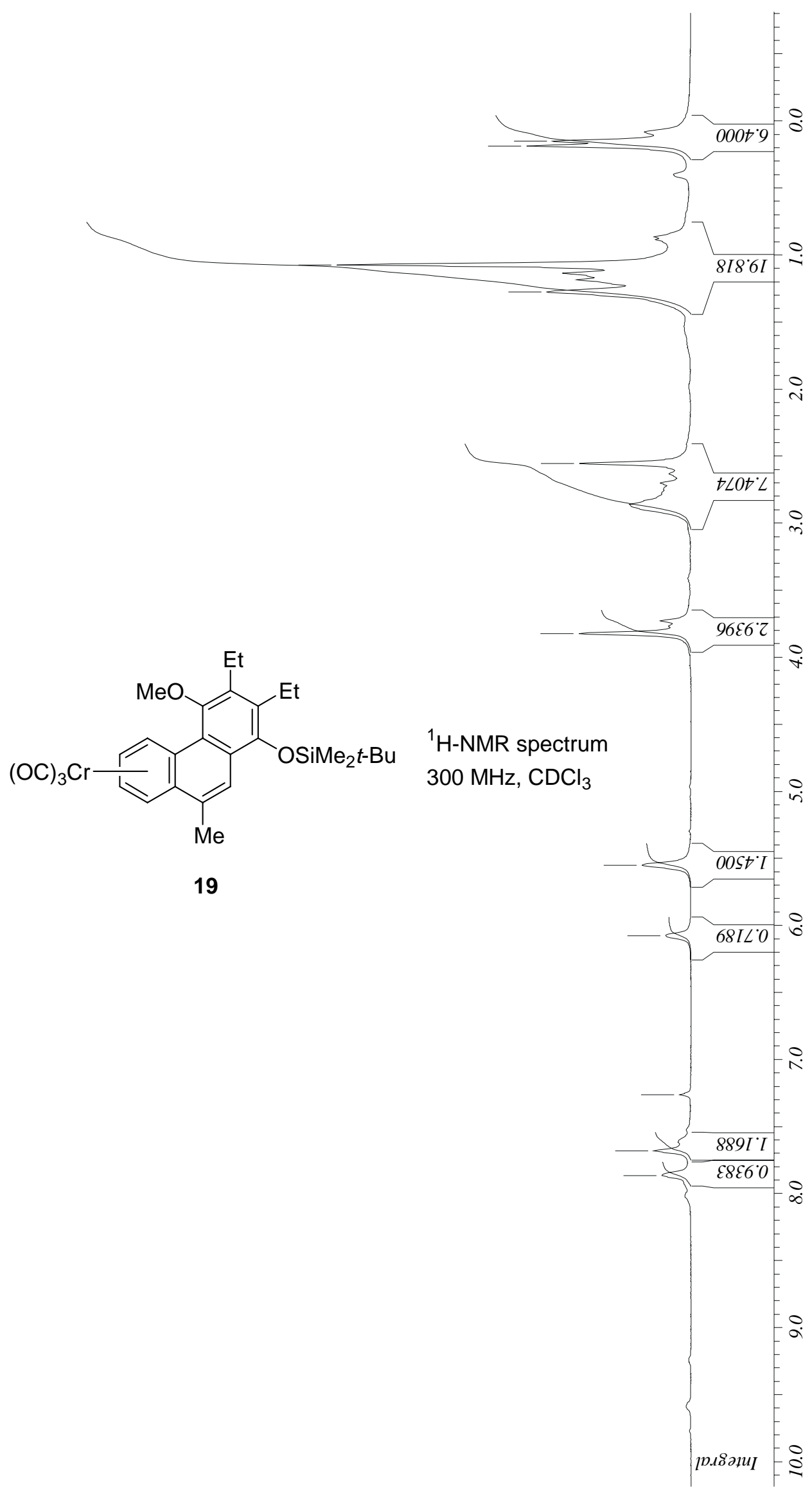

$0092: \angle$

$5089^{\circ} \mathrm{L}$

$0 S \angle 0^{\circ} 9$ 乙E98 $\angle$ 
${ }^{\circ} 09 \varepsilon^{\circ} \varepsilon^{-}$

$6{ }^{\circ} \mathrm{CZ}^{\circ} \mathrm{E}-$

$8065^{\circ} \triangleright I$

SIS8 $S I$

II08 8 I

$6609^{\circ} 6 I$

IE9 ${ }^{\circ} 0 \mathrm{C}$

$68 \varepsilon 8^{\circ} 0 乙$

$\angle E O I^{\circ} 9 Z$

ZISt' I9

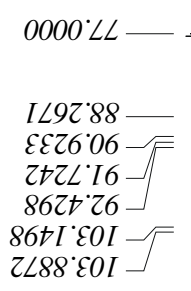

I $60^{\circ} 0 Z I-$
SS8I'tZI

SS8I" $\nabla 2 I$

66IL $6 Z I$

$6 \varepsilon I \nabla \circ \varepsilon I$

S808'9EI

$t \varepsilon 98^{\circ} \mathrm{S} t I$

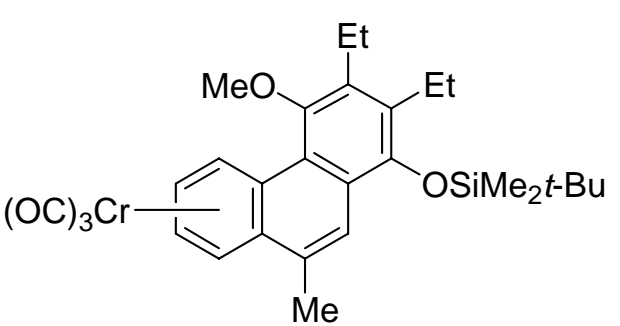

19

EICZ

It60.0ZI

$S S 8 I^{\circ} \hbar Z I$

$\angle 8 E 6^{\circ} \circ Z I$

66IL'6ZI

$6 \varepsilon I t^{\circ} t \varepsilon I$

S808:9EI _

o

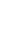


$z \varepsilon \varsigma \varsigma \cdot$

$t 8 t \varsigma^{\circ} \varsigma$

$0 S \angle 0^{\circ} 9$

$0092^{\circ} \mathrm{L}$

$5089^{\circ} \mathrm{L}$

ZE9 $8^{\circ} \mathrm{L}$

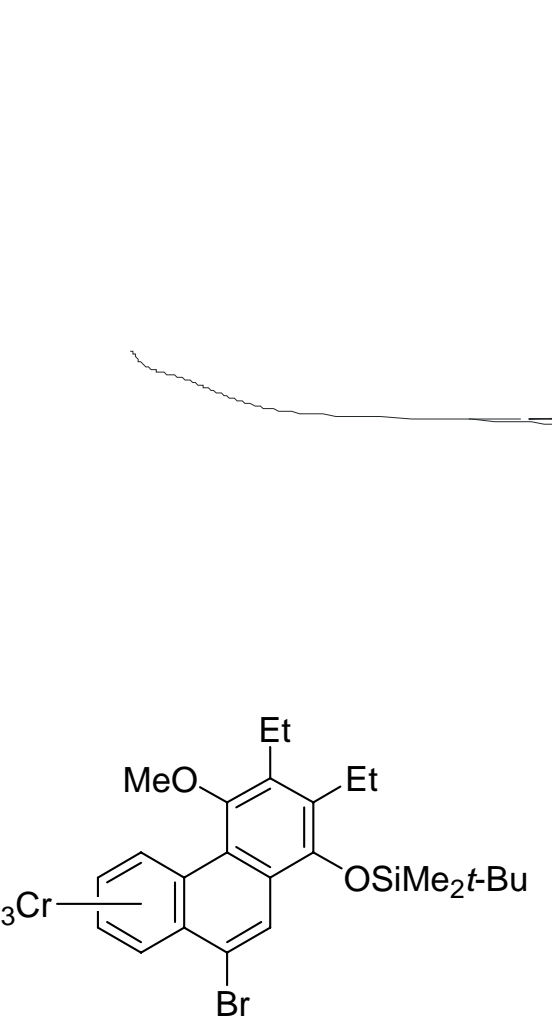

20
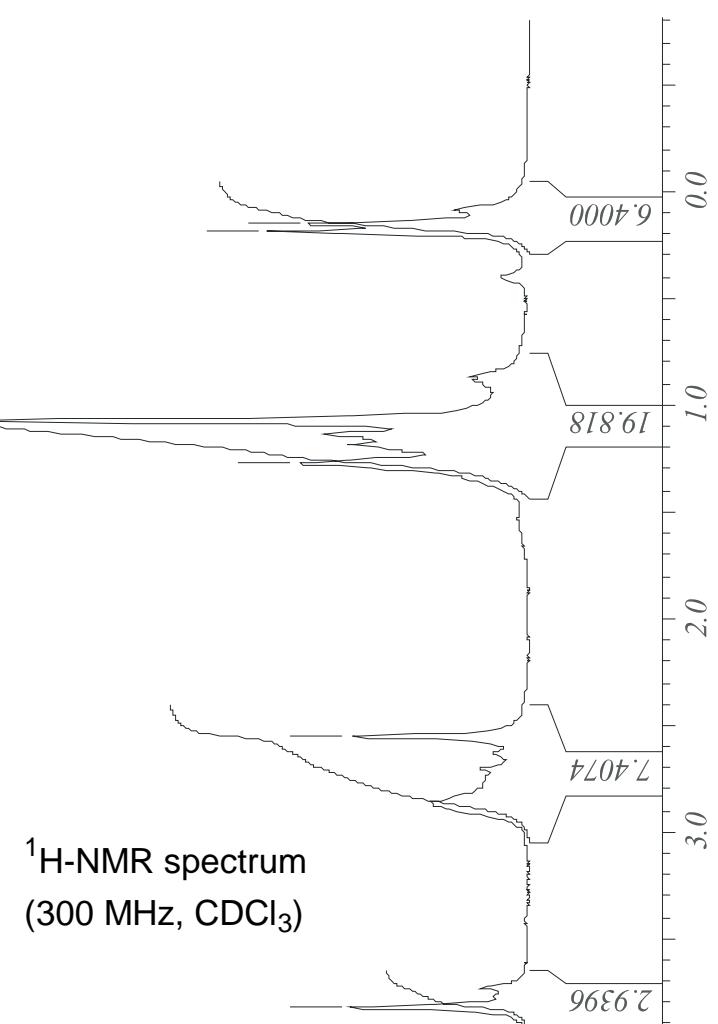
t09E $\mathcal{E}^{-}$

$610 z^{\circ} \mathcal{E}$

$806 c^{\circ} t$

SIS $8^{\circ}$ SI

II08 81

660961

IE9E 02

$68 \varepsilon 8^{\circ} 0 \mathrm{Z}$

$\angle \mathcal{E} O I^{\circ} 9 \mathrm{Z}$

zISt 19

$0000^{\circ} L L$

I $\angle 927^{\circ} 88$

EEZ6.06

Z†ZL'I6

$862 t \cdot 26$

$86+I^{\circ} \mathcal{E} 0 I$

Z $\angle 88^{\circ} \mathcal{E} 0 \mathrm{I}$

It $60.02 I$

$\varsigma \varsigma 8 I^{\circ}+2 I$

$\angle 8 E \sigma^{\circ} \circ Z I$

66IL $62 I$

$6 \varepsilon I+\circ \varepsilon I$

S808 $9 \mathcal{E} I$

$t \varepsilon 98^{\circ} S t I$

$\mathcal{E} I \mathcal{E} Z$ ISI

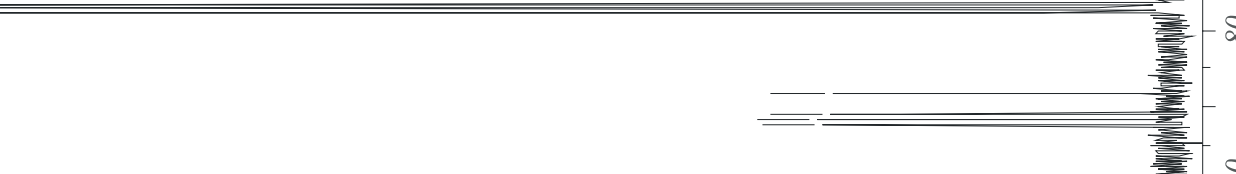

It60 0ZI

$6 \varepsilon I+t) E L$

S808.9EI _

6SI6 ZEZ<smiles>CCc1c(CC)c(OCC(C)(C)C)c2c(cc(Br)c3cc(Cl)ccc32)c1OC</smiles>

${ }^{13} \mathrm{C}-\mathrm{NMR}$ spectrum

(75 $\mathrm{MHz} \mathrm{CDCl}_{3}$ )

8

ฐ

$\frac{2}{\sqrt[2]{2}}$

$\stackrel{8}{8}$

$s S 8 I \circ Z I$

$\angle 8 E \sigma^{\circ}+Z I$

$66 I L 62 I$

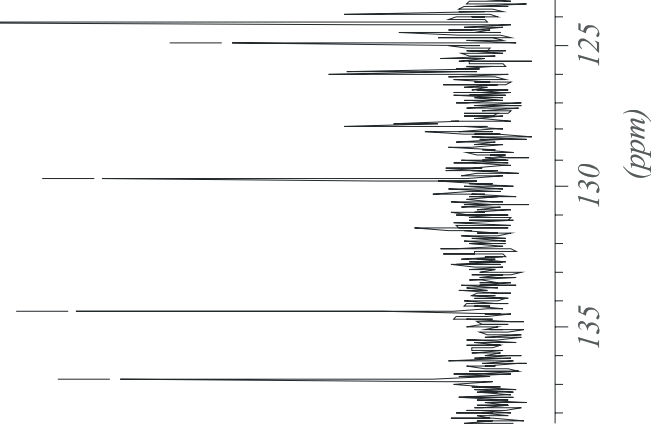

$\infty$

$\stackrel{2}{8}$

สิ

우

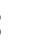




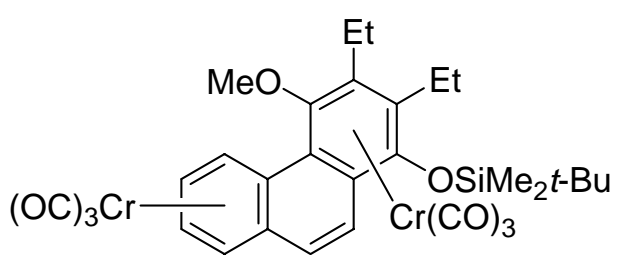

${ }^{1} \mathrm{H}$-NMR spectrum -1.5 to $4.5 \mathrm{ppm}$ (300 MHz, $\mathrm{CDCl}_{3}$ )

$9 Z \angle 0^{\circ} 0$

$8 D \angle Z^{\circ} 0$

$2662^{\circ} 0$

22

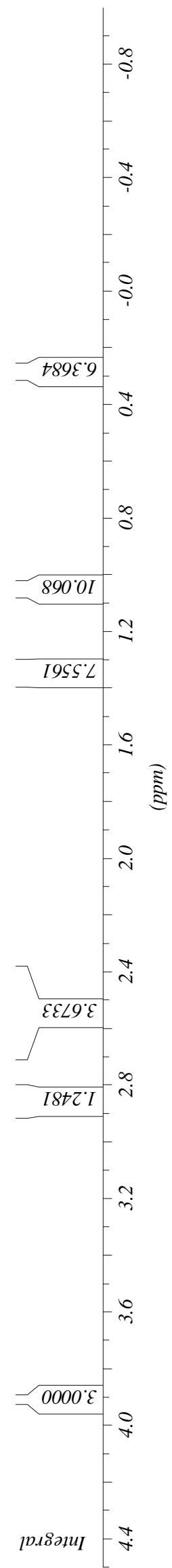

$6 t 50^{\circ}$

$9 I I E^{*} I$

$982 \mathcal{E}^{\circ} I$

IESE $I$

乙I9E' $I$

乙 $\angle E^{\circ} I$

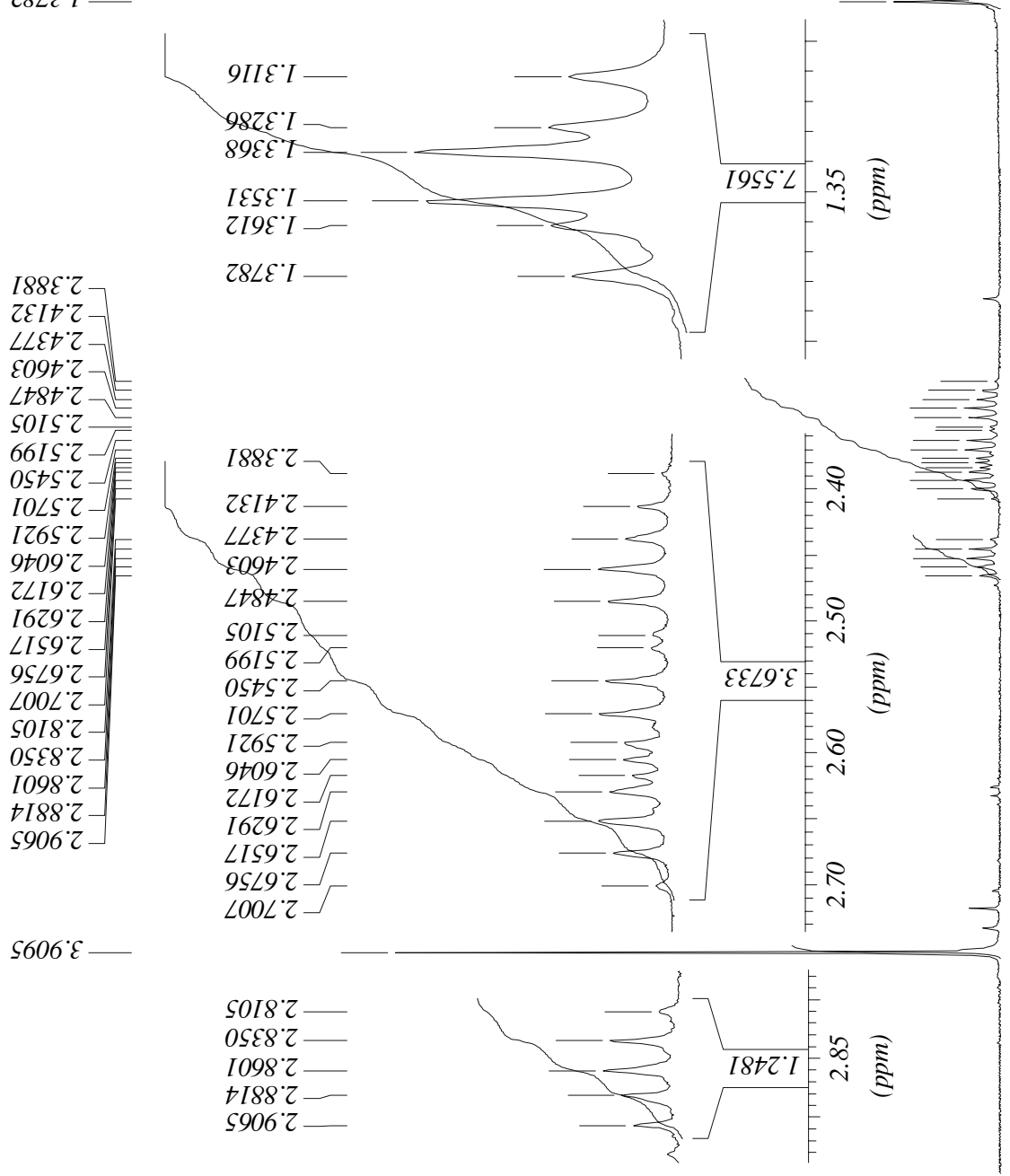




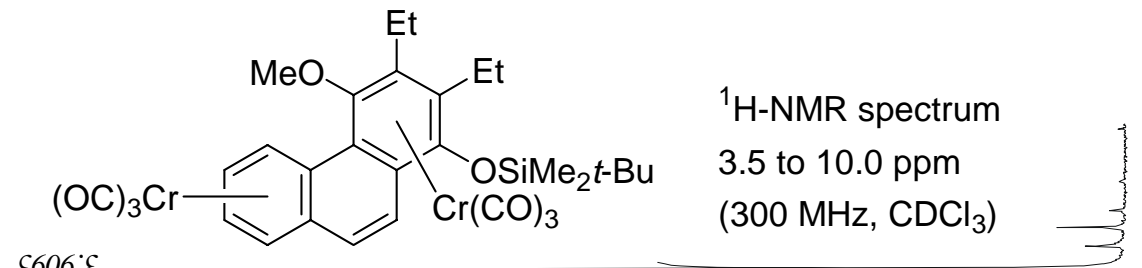

$5606^{\circ} \varepsilon$

$$
\begin{aligned}
& S E t t^{\circ} S-22 \\
& 6 \angle t D^{\circ} S- \\
& 989 t^{\circ} S- \\
& 089 t^{\circ} S-[ \\
& \left.Z I \angle D^{\circ} S\right] \\
& \left.S \angle 8 t^{\circ} S\right]
\end{aligned}
$$

Z86L'S

$\nabla Z \angle L \cdot S$

$89 \angle L \cdot S$

$8 \varepsilon 6 \angle L^{\circ} \mathrm{S}$

$286 L^{\circ} \mathrm{S}$

$6 S t S^{\circ} S$

$06+S^{\circ} S \longleftarrow$

ZL9S'S

EOLG $S_{-}$

$6 \angle 8 S^{\circ} S$

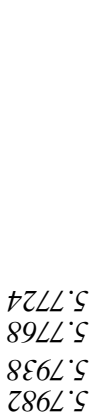

.

$5678 \cdot 9$

$6088^{\circ} 9$

$009 Z^{\circ} \angle$

EI8Z $\angle-$

$9+0 \varepsilon^{\circ} L$

9zZt $\angle$

$9 t S t^{\circ} L$

$56 t 8{ }^{\circ} 9$

$6088^{\circ} 9$
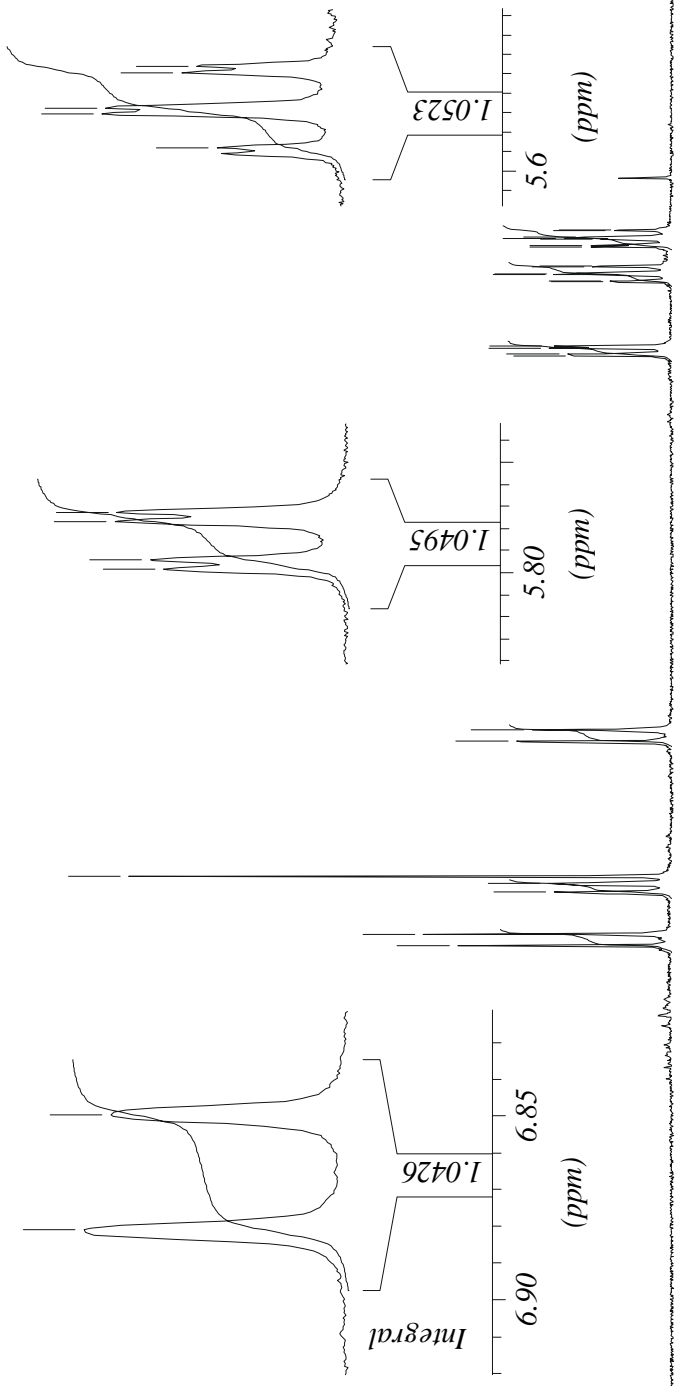

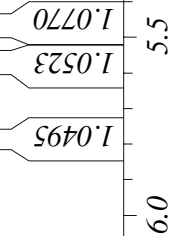

हิ

$28 t 0 \cdot \mathrm{I}$

$690 I^{\circ} \mathrm{I}$

EI8Z: $\angle$

$9+0 \varepsilon^{\circ} \angle$

$9 z 25^{\circ} L$

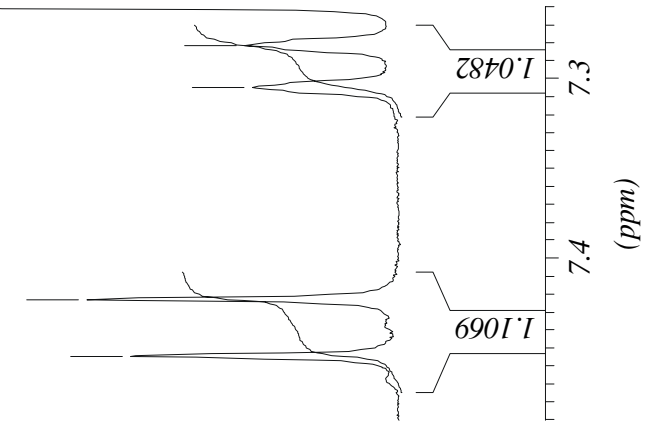

is

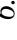

?

$\stackrel{\circ}{\circ}$

$\stackrel{2}{\wedge}$

$\stackrel{\infty}{\infty}$

$L_{\infty}^{\infty}$

$\stackrel{\circ}{+}$

$\stackrel{\leftrightarrow}{+}$

है

$\stackrel{\circ}{\circ}$

$\stackrel{5}{9}$ 
I8t6 $6^{\circ} \mathrm{C}^{-}$

$0 \downarrow \varepsilon \angle Z^{\circ} Z^{-}-$

$\downarrow 800^{\circ} \mathrm{I}$

$\triangle I 0 Z^{\circ} S I$

$98 \varepsilon \angle{ }^{\circ} \angle I$

SZL8 81

I808.6I

$\angle L I D^{\circ} I Z-$

$(\mathrm{OC})_{3} \mathrm{Cr}$

${ }^{13} \mathrm{C}$-NMR spectrum

(75 MHz, $\mathrm{CDCl}_{3}$ )

$8 \angle t \angle 99$

22

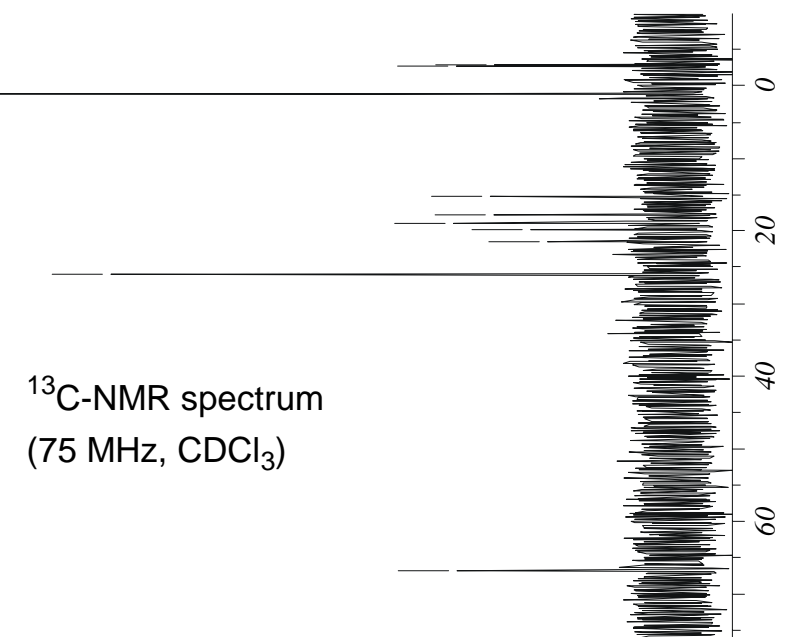

$0000^{\circ} \mathrm{LL}$

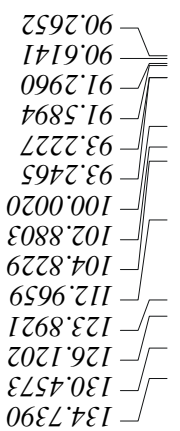

乙S92.06

It I9.06-

096 ${ }^{\circ}$ I6 -

${ }^{\circ 68 S^{\circ}} \mathrm{I6}$

$\angle Z Z Z^{\circ} \varepsilon 6$

s9t $\tau^{\circ} \varepsilon 6-$

$0 Z 00^{\circ} 00 \mathrm{I}$

E088'Z0I

6ZZ8

6S96 ZII

IZ68. $E Z I$

Z0ZI'9ZI

$\varepsilon \angle S t^{\circ} 0 E I$

$06 \varepsilon \angle{ }^{\circ} t \varepsilon I$

$\pitchfork S \angle 0^{\circ} Z E Z$

S†

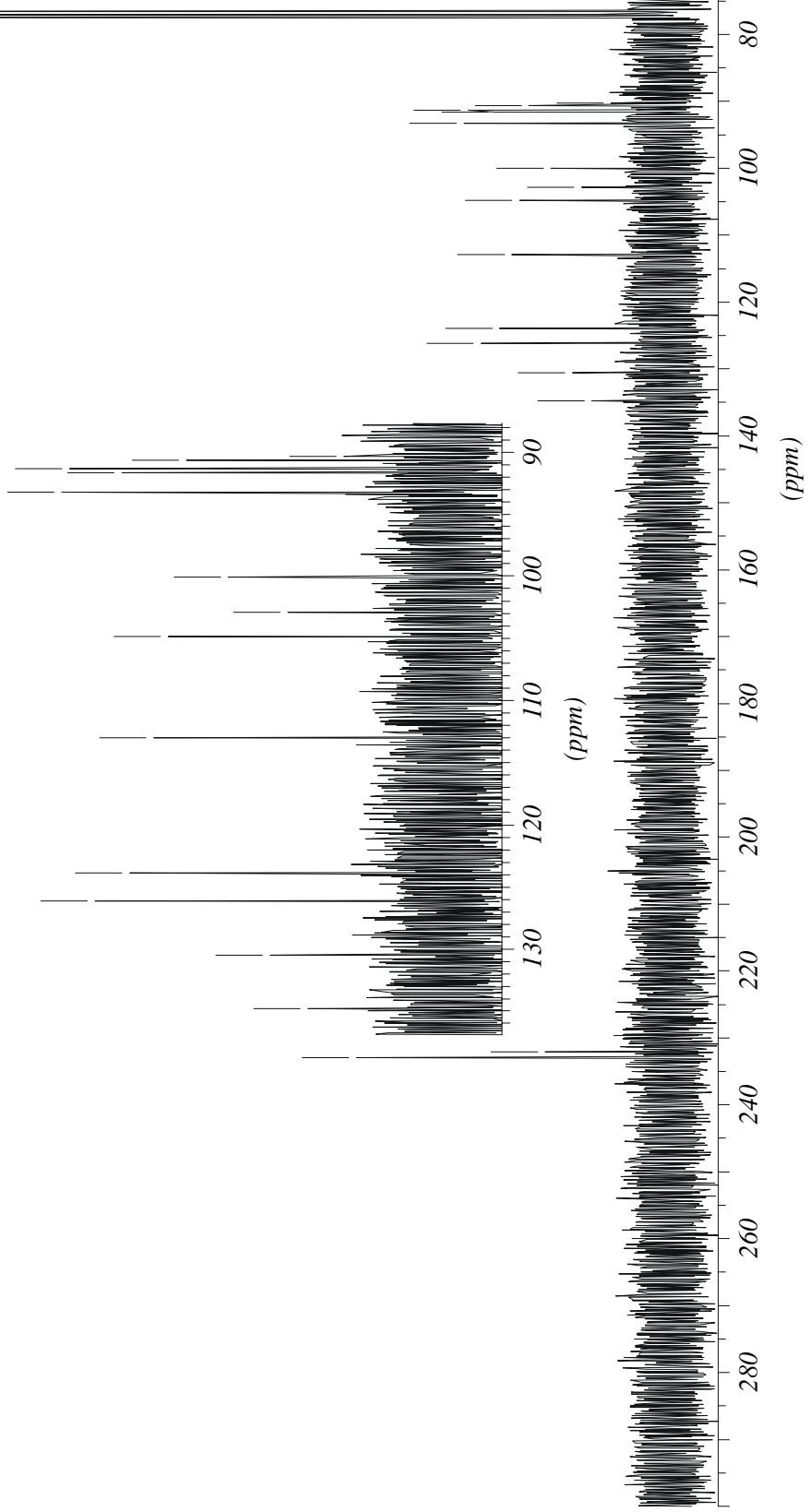

